\title{
Avaliação de Dados Aeromagnetométricos na Porção Oeste de Roraima a partir de técnicas de processamento digital em ambiente SIG.
}

Phaulla Oliveira, Solange dos Santos Costa, Igor da Silva Jacaúna.

Copyright 2019, SBGf - Sociedade Brasileira de Geofísica

This paper was prepared for presentation during the $16^{\text {th }}$ International Congress of the Brazilian Geophysical Society held in Rio de Janeiro, Brazil, 19-22 August 2019.

Contents of this paper were reviewed by the Technical Committee of the $16^{\text {th }}$ International Congress of the Brazilian Geophysical Society and do not necessarily represent any position of the SBGf, its officers or members. Electronic reproduction or storage of any part of this paper for commercial purposes without the written consent of the Brazilian Geophysical Society is prohibited.

\section{Abstract}

The research covers the western portion of Roraima, NA.20-V-D sheet in the Parima-Uraricoera Domain, based on the interpretation and integration of aerogeophysical signatures with available geological data. Data corresponding to the Parima-Uraricoera Aerogeophysical Project and corresponding shapefiles of the study area, made available by the Geological Survey of Brazil, were used. The digital processing was carried out using the Oasis Montaj software, where the image of the Residual Magnetic Field (MAGR) was generated, and the Analytical Signal Amplitude (ASA) filter was applied, which is linked to the magnetization amplitude and enabled to delimit the sources present. For the mapping, it was necessary to apply digital processing techniques in a geographic information system (GIS) environment, based on the classification not supervised by means of the ISODATA algorithm, then the vectorization and extraction of the classes (major and minor intensities magnetic fields). A comparative analysis was made between the classified image and the geological data, obtaining the map of aeromagnetic domains. As a result, it was identified that the units with the highest intensity are associated with mafic and metamorphic igneous units, such as the Máfica and Ultramafic Suite Serra da Estrutura, and the units with lower intensity correspond to sedimentary lithotypes, such as the Roraima Supergroup.

\section{Introdução}

A área de estudo abrange o Domínio Parima-Uraricoera (FRAGA et al., 2008), , porção Oeste do Estado de Roraima, com fronteira com a Venezuela (Figura 1), correspondente a Folha NA.20-V-D. (Serra Urucuzeiro), situada na porção oeste do estado de Roraima, entre as longitudes $63^{\circ} 00^{\prime} 00^{\prime \prime} \mathrm{W}$ e $64^{\circ} 22^{\prime} 00^{\prime \prime} \mathrm{W}$ e latitudes $02^{\circ} 00^{\prime} 00^{\prime \prime} \mathrm{N}$ e $03^{\circ} 00^{\prime} 00^{\prime \prime} \mathrm{N}$, inserida em território indígena e caracterizada por uma densa vegetação, tornando o mapeamento escasso e com pouco conhecimento geológico. Os dados aerogeofísicos são ferramentas que permitem o reconhecimento de feições geológicas por meio de padrões das anomalias que permitem 0 mapeamento e interpretação de dados e a investigação geológica da área de interesse. A utilização de dados aerogeofísicos tem sido utilizada com sucesso em pesquisas geológicas na Amazônia. (OLIVEIRA et al., 2017).
O Sensoriamento Remoto, segundo Meneses e Almeida (2012, p.3) é definido como a técnica de obter imagens de áreas da superfície terrestre e o processamento digital consiste em técnicas que visam a manipulação dos dados para melhorar o aspecto visual de feições e facilitar a extração de informações (NASCIMENTO et al. 2012). Dentro das diversas técnicas existentes, a classificação é uma ferramenta de grande utilidade, baseada na extração de informação em imagens com a finalidade de reconhecer padrões e objetos homogêneos, sendo utilizados no mapeamento de áreas de diversos temas de interesse (FIGUEIREDO, 2005).

Desta forma, o objetivo desta pesquisa é a avaliação de dados aeromagnetométricos utilizando técnicas de processamento digital em ambiente SIG e verificação da eficácia da classificação de dados aerogeofísicos para a região de estudo.

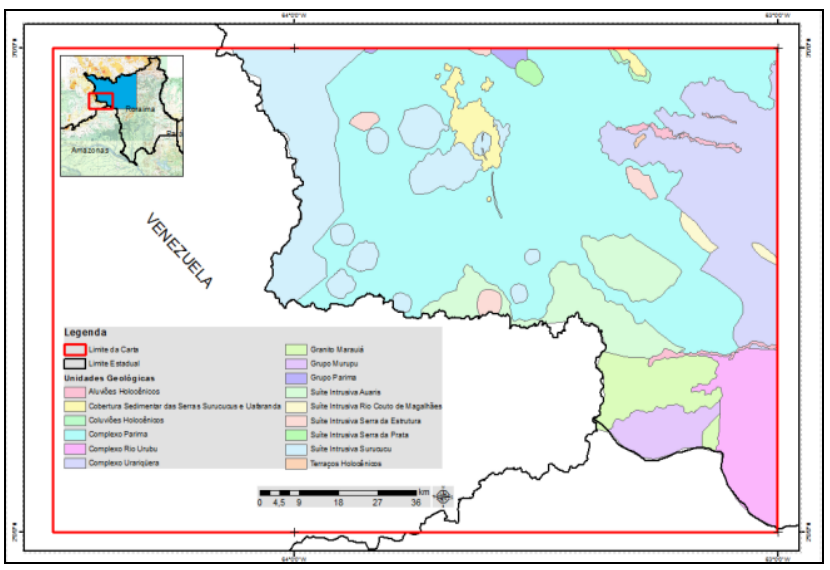

Figura 1. Mapa Geológico da Folha NA.20-V-D, (cor vermelha)

\section{Metodologia}

Os dados aerogeofísicos utilizados foram disponibilizados pelo Serviço Geológico do Brasil, correspondente ao Projeto Aerogeofísico 1058- Província Mineral ParimaUraricoera, com Linhas de Voo (LV) de 0,5 km e de controle de $10 \mathrm{~km}$, com orientação NS e EW e com altura de voo de $100 \mathrm{~m}$ (CPRM, 2001).

Estes insumos foram processados com base nas ferramentas fornecidas pelos programas ArcGis 10.4.1 e Oasis Montaj 6.3, e ENVI 5.3. As etapas da metodologia encontram-se resumidas na figura 2 , e detalhadas nos itens a seguir: 


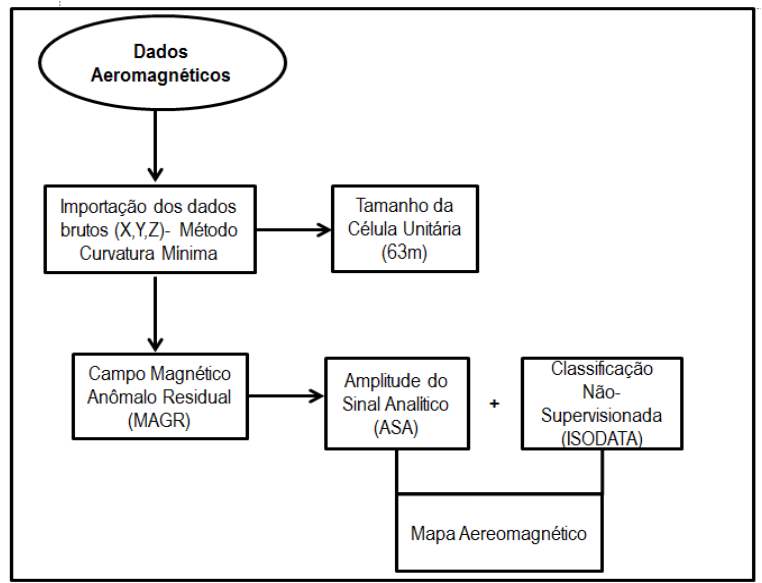

Figura 2. Etapas dos procedimentos metodológicos.

Para a pesquisa, foram utilizados os dados brutos em formato $X, Y, Z$ que permite o processamento dos dados desde as etapas iniciais. Para tanto, foi utilizado o software Oasis Montaj 6.3, os dados foram importados e interpolados pelo método de Curvatura Mínima, (KECKLER, 1994). Por seguinte foi feita a definição da célula unitária de $1 / 4$ do espaçamento entre as linhas de voo $(500 \mathrm{~m})$ que equivale ao valor arredondado de $63 \mathrm{~m}$. Foi gerada a imagem do Campo Magnético Residual (MAGR) e posteriormente foi aplicado na Transformada Rápida de Fourier, o filtro de Amplitude do Sinal Analítico (ASA) e que permiteu a delimitação das fontes magnéticas (MILLIGAN e GUNN (1997), BLUM (1999)).

Foi utilizado o histograma gerado por Dobrin e Savit (1988) e modificado por Oliveira et al. (2017), para a avaliação das respostas das intensidades identificadas na imagem de ASA, neste pode-se observar a correlação dos minerais magnéticos e litologias, onde os valores mais altos de susceptibilidade correspondem á rochas ígneas básicas, enquanto rochas sedimentares vão apresentar valores mais baixos (Figura 3).

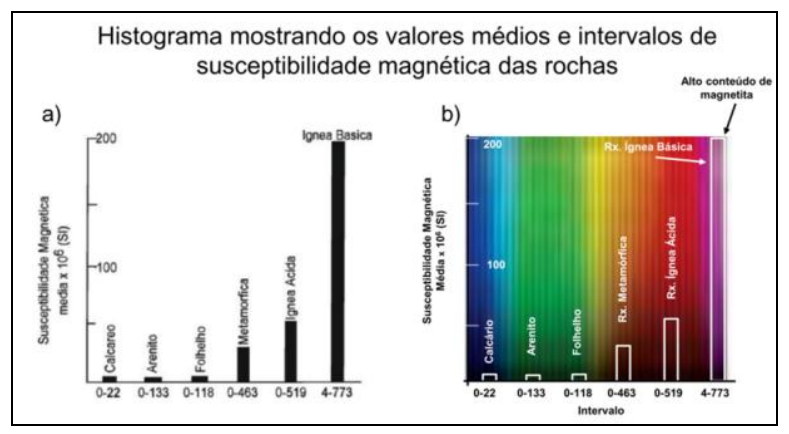

Figura 3. Histograma de Susceptibilidade Magnética, a) baseado em Dobrin e Savit (1988); b) Histograma modificado por Oliveira et al. (2018).

Para identificar e mapear as concentrações de minerais magnéticos na área de estudo os dados raster foram importados para o software ENVI 5.3, foi utilizado o classificador não supervisionado ISODATA, sendo determinado no mínimo 5 e no máximo 11 classes. A acurácia da classificação foi de acordo com o método estatístico índice Kappa (Tabela 1). Ao final, a imagem classificada foi vetorizada e extraída apenas as classes de interesse, ou seja, maiores e menores áreas de concentração de minerais magnéticos.

Tabela 1. Qualidade da classificação versus valores estatísticos Kappa. Adaptado de Landis e Koch (1977).

\begin{tabular}{c|c}
\hline Valor de Kappa & Qualidade do mapa temático \\
\hline$<0,00$ & Péssima \\
\hline $0,00-0,20$ & Ruim \\
\hline $0,20-0,40$ & Razoável \\
\hline $0,40-0,60$ & Boa \\
\hline $0,60-0,80$ & Muito Boa \\
\hline $0,80-100$ & Excelente
\end{tabular}

\section{Resultados}

Foi observado no Campo Magnético Residual (MAGR) em pseudocor apresentou valores do campo magnético que se encontram entre - 2265,1 nT e 1637,7 nT, a cor magenta representa os valores máximos e a cor azul representa os valores mínimos (Figura 4). Observa-se que as anomalias magnéticas apresentam o indício de inúmeras feições estruturais alongadas, que foram melhor definidas a partir da Amplitude do Sinal Analítico (Figura 5). Nesta imagem, podem-se observar várias fontes com valores magnéticos elevados, provavelmente associados às rochas máficas e ultramáficas existentes na área.

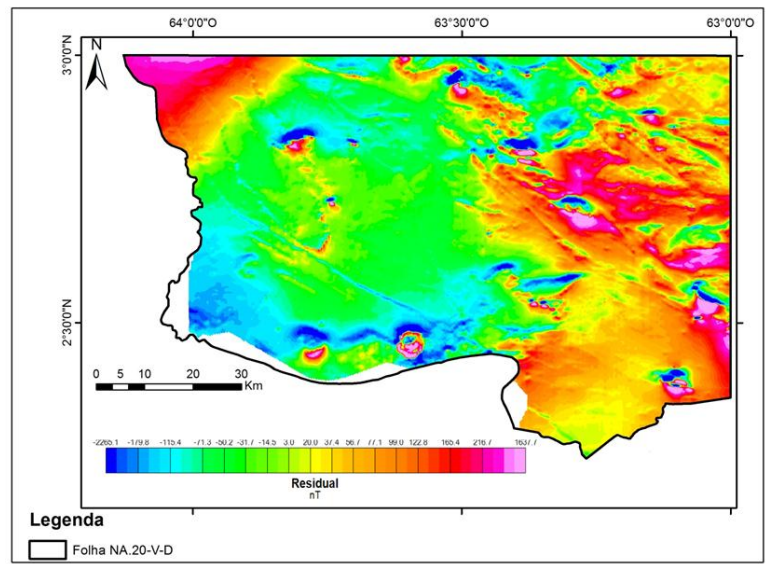

Figura 4. Campo Magnético Anômalo Residual (MAGR), obtido a partir do método de Curvatura Mínima.

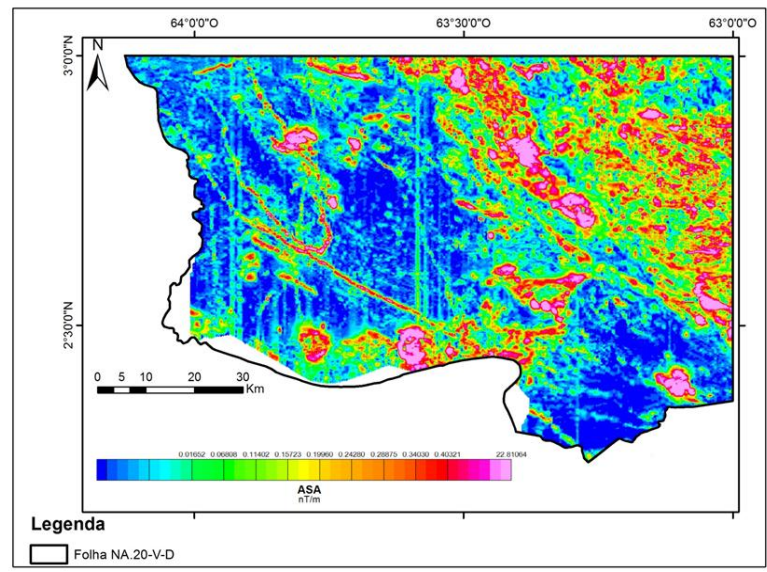

Figura 5. Imagem da Amplitude do Sinal Analítico (ASA). 
Com base na imagem da Amplitude do Sinal Analítico foi possível identificar as áreas com maior e menor concentração de minerais magnéticos e correlacioná-los com a litologia.

A imagem classificada através do algoritmo ISODATA, gerou 11 classes, sendo extraídas apenas as duas classes de interesse (concentrações máximas e mínimas). A mensuração da acurácia da classificação por intermédio do método estatístico Kappa forneceu um valor de 0,97, que de acordo com a tabela de índice Kappa proposto por Landis e Koch (1977), a média de acurácia e a qualidade do mapa temático obtidos correspondem ao índice excelente.

Como resultado extração, obteve-se uma imagem vetorizada (Figura 6), na qual as áreas em azul escuro correspondem à rochas que apresentam baixas concentrações magnéticas, enquanto as áreas em magenta estão relacionadas à rochas que apresentam altas concentrações magnéticas.

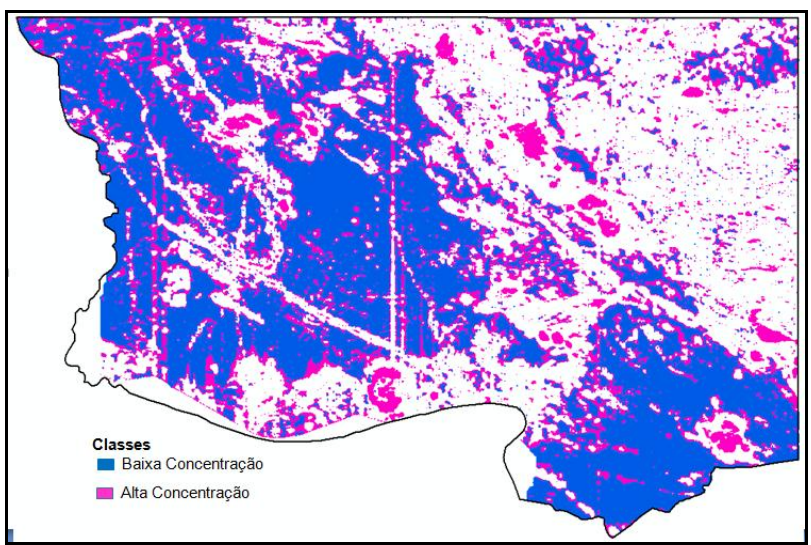

Figura 6. Resultado da extração das classes, em azul escuro, indicando baixas concentrações magnéticas, e magenta, representando altas concentrações magnéticas.

Posteriormente, foi realizada a comparação entre a imagem classificada com os dados vetoriais do mapa geológico, onde foi possível gerar o mapa de domínios magnéticos (Figura 7).

Os valores mais altos das concentrações de minerais magnéticos estão associados com os litotipos Suíte Máfica e Ultramáfica Serra da Estrutura (rochas máficas ígneas e metamórficas), Suíte Intrusiva Surucucus (rochas ígneas básicas), Grupo Parima (Metavulcânicas, Paragnaisses e Quartzitos), Suíte intrusiva Auaris (rochas ígneas intermediárias) e Suíte Intrusiva Ericó (rochas ígneas básicas).

Já as concentrações de mais baixo valor se correlacionam com os litotipos Supergrupo Roraima (cobertura sedimentar), Grupo Parima (Paragnaisses e Quartzitos), Complexo Uraricoera (rochas ígneas intermediárias) e Suíte Intrusiva Auaris (rochas ígneas intermediárias).

A partir da análise dos valores do histograma de susceptibilidade magnética (Figura 3), pode-se correlacionar as altas concentrações identificadas na imagem, como parte da análise selecionou-se uma porção da imagem correspondente a Suíte Máfica e
Ultramáfica Serra da Estrutura, ao qual é constituída por litotipos como gabronoritos, gabros e Iherzolitos. Em seguida, selecionou-se outra porção da imagem, que corresponde ao Supergrupo Roraima, que é formada por rochas sedimentares, desta forma, ao analisar esta região observam-se valores de baixas concentrações de minerais magnéticos, notado na Figura 7.

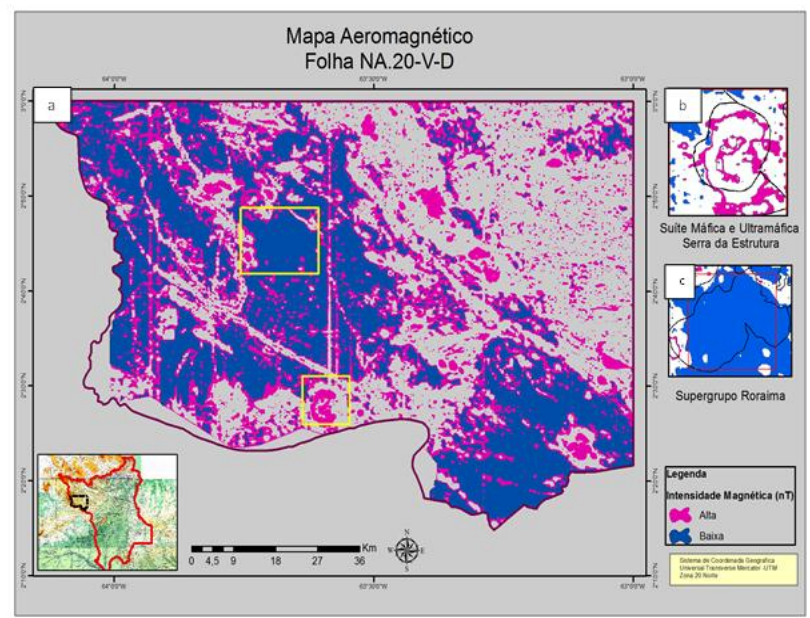

Figura 7. Sobreposição da imagem classificação com o mapa geológico, as baixas e altas intensidades magnéticas (em azul e magenta, respectivamente). Em destaque: b) Suíte Máfica e Ultramáfica Serra da Estrutura (polígono preto) indicando altas concentrações de minerais magnéticos; c) Supergrupo Roraima (polígono preto) evidenciado por baixas concentrações de minerais magnéticos.

\section{Conclusões}

A imagem da Amplitude do Sinal Analítico, obtido a partir dos dados aeromagnetométricos permitiu a identificação de duas classes magnéticas. Estas integradas ao mapa geológico permitiu efetuar a correlação entre a alta ou baixa intensidade magnética e os litotipos mapeados, validado pelos dados vetoriais disponíveis. Os dados aeromagnéticos viabilizaram a comparação com os dados vetoriais geológicos, o que permitiu propor um mapa aeromagnético que poderá servir como ferramenta, juntamente á integração de outros tipos de insumos, para a atualização dos mapas e cartas disponíveis da região de estudo.

\section{Agradecimentos}

Ao Centro Regional de Manaus do Sistema de Proteção da Amazônia - SIPAM, por oferecer toda a estrutura necessária para desenvolvimento desta pesquisa através do Programa de Apoio de Iniciação a Pesquisa - PAIC e a Fundação de Amparo à Pesquisa do Estado do Amazonas pelo financiamento da bolsa de estudo e pela oportunidade dada para a realização desse trabalho.

\section{Referências}

BLUM M.L.B. Processamento e Interpretação de Dados de Geofísica Aérea no Brasil Central e sua Aplicação a Geologia Regional e a Prospecção Mineral. Tese de Doutorado, Instituto de Geociências, Universidade de Brasılia, 229 p,1999 
CPRM. Projeto Aerogeofísico Província Mineral Parima-

Uraricoera. Relatório final do levantamento e gamaespectrométricos. Brasília: CPRM, 2001.

FIGUEIREDO, D. Conceitos Básicos de Sensoriamento Remoto. CONAB, 2005.

DOBRIN M.B. e SAVIT C.H. Introduction to Geophysical Prospecting. McGraw-Hill Book Co., New York, 867 p. 1988.

FRAGA L.M.B., REIS N.J., DALL'AGNOL R., ARMSTRONG R. Cauarane-Coeroeni Belt - The tectonic southern limit of the preserved Rhyacian crustal domain in the Guyana Sheild, northern Amazonian craton. In: Int. Geol. Cong., 33, Oslo, Noruega. CD-Rom, 2008.

LANDIS, J.R. e KOCH, G.G. The measurement of observer agreement for categorical data. Biometrics, v.33, n.1, p. 159-174, 1977.

KECKLER, D.Surfer for Windows. User's Guide.Golden Software Inc, Golden, CO, USA, 450 p,1994.

MENESES, P. R.; ALMEIDA, T. Introdução ao Processamento de Imagens de Sensoriamento Remoto. Brasília, 2012. 276p.

MILLIGAN P.R.; GUNN P.J. Enhancement and presentation of airborne geophysical data. AGSO JournalofAustralianGeology\&Geophysics, 17(2): 63-75. 1997.

NASCIMENTO, R. F. F.; Alcântara, E. H.; Kampel, M.; Stech, J. L.; Novo, E. M. L. M.; Fonseca, L. M. G. O Algoritmo Support Vector Machines (SVM): Avaliação da Separação Ótima de Classes em Imagens CCD-CBERS2. In: Simpósio Brasileiro de Sensoriamento Remoto, 14, 2009, Natal, Brasil. Anais... São José dos Campos: INPE. p. 2079-2086.2009

OLIVEIRA, P., COSTA S.S., COSTA J. A. L. SALAZAR C. A. Caracterização geológica da porção oeste de Roraima a partir de Imagens R99/SAR, dados SRTM e aeromagnéticos. Projeto de Iniciação Científica - PAIC. Sistema de Proteção da Amazônia, 2017. 